\title{
Retrospective Study of Trazodone Monotherapy Compared with Ramelteon and Trazodone Combination Therapy for the Management of Delirium
}

Takao Ishii $^{1 *}$, Takafumi Morimoto ${ }^{2}$, Masaki Shiraishi ${ }^{1}$, Yoshiyasu Kigawa ${ }^{1}$, Kenji Narita ${ }^{1}$, Keisuke Inoue ${ }^{3}$ and Chiaki Kawanishi $^{1}$

${ }^{1}$ Department of Neuropsychiatry, School of Medicine, Sapporo Medical University, Sapporo, Japan

${ }^{2}$ Department of Occupational Therapy, School of Health Sciences, Sapporo Medical University, Sapporo, Japan

${ }^{3}$ Psychiatric Center, Yokohama City University Medical Centre, Yokohama, Japan

\begin{abstract}
Background: Accumulating evidence supports the efficacy of antipsychotics in patients with delirium. However, extrapyramidal symptoms caused by antipsychotic medications represent a major concern in the pharmacotherapy of delirium, meaning that therapeutic alternatives using drugs other than antipsychotics are required. The aim of this study was to compare the efficacy and tolerability of trazodone monotherapy with ramelteon and trazodone combination therapy for the management of the symptoms of delirium.
\end{abstract}

Methods: A case-control study was conducted in a general hospital setting. Delirium Rating Scale-revised-98 (DRS-R-98) scores were measured at the initial examination and at 3-7 days after starting the study drugs. Adverse drug reactions were also assessed.

Results: Thirty-three patients were retrospectively enrolled to the trazodone monotherapy group (T group) and 59 patients were enrolled to the ramelteon and trazodone combination therapy group (RT group). No significant differences in demographic characteristics were observed between the groups. Following treatment, the total DRS-R-98 scores were significantly reduced in both groups $(22.0 \pm 5.5$ to $13.5 \pm 8.5$ in the T group and $23.7 \pm 6.1$ to $11.4 \pm 8.6$ in the RT group). However, the proportion of patients meeting the remission criteria was significantly higher in the RT group than in the T group $(71 \%$ vs. $48 \%$; chi-square $=4.681, p=0.030)$. The most commonly reported side effect was somnolence, in the RT group (3\%).

Conclusion: Our findings indicate that both trazodone monotherapy and combination therapy of ramelteon and trazodone were effective in managing the symptoms of delirium. However, more patients met the remission criteria following combination therapy with ramelteon and trazodone. In both groups, the incidence of adverse drug reactions was very low.

Keywords: Delirium; Ramelteon; Trazodone; Pharmacotherapy

\section{Introduction}

Delirium is a common condition characterized by an acute disturbance in attention and awareness, which is caused by an underlying medical disorder. Highly prevalent among medical hospitalized patients [1,2], delirium imposes a considerable burden on patients because of the increased morbidity and mortality that it is associated with $[3,4]$. Therefore, early management of this condition may lead to better health outcomes.

Few studies have examined the pathophysiology of delirium, and the condition is therefore not well understood. Recent evidence suggests that an imbalance in neurotransmitters, particularly reduced cholinergic function, excess release of dopamine, or both, may cause the various symptoms of delirium [5-7]. Thus, antipsychotic medications such as haloperidol, olanzapine, and risperidone are commonly used to control delirium symptoms based on the majority of clinical guidelines for agitated delirium $[8,9]$. To date, however, conclusive evidence of the effectiveness of pharmacological prevention or treatment of delirium is lacking [2,10]. In addition, given that patients with delirium are typically in poor physical condition, antipsychotics can increase the risk of adverse drug reactions such as extrapyramidal symptoms, sedation, drowsiness, neuroleptic malignant syndrome, and QT interval prolongation [11-15]. These side effects of antipsychotics can delay the physical recovery of patients and increase their mental or physical discomfort.

Trazodone, a heterocyclic antidepressant, is frequently used to treat insomnia in patients with and without depression [16,17], and is also used in Japan to treat insomnia in patients with delirium. To date, one study has described the effect of trazodone on delirium; Okamoro et al. reported that seven cases of delirium had improved following treatment with trazodone as add-on to benzodiazepine or haloperidol [18]. However, no studies to date have reported the effects of trazodone monotherapy or trazodone in combination with other types of hypnotics on delirium. Ramelteon, a melatonin receptor agonist, has been used for the treatment of insomnia characterized by difficulty with sleep onset. Although no large studies have been reported to date, recent research indicates that ramelteon is an effective option for the treatment or prevention of delirium [19-21].

The aim of this preliminary study was to investigate the efficacy and tolerability of trazodone monotherapy and ramelteon and trazodone combination therapy on the management of delirium symptoms,

*Corresponding author: Takao Ishii, Department of Neuropsychiatry, School of Medicine, Sapporo Medical University, Sapporo, Japan, Tel: +81116112111; Fax +81116443041; E-mail: ishitaka@sapmed.ac.jp

Received: February 28, 2018; Accepted: April 05, 2018; Published: April 15 2018

Citation: Ishii T, Morimoto T, Shiraishi M, Kigawa Y, Narita K, et al. (2018) Retrospective Study of Trazodone Monotherapy Compared with Ramelteon and Trazodone Combination Therapy for the Management of Delirium. J Psychiatry 21: 444. doi:10.4172/2378-5756.1000444

Copyright: ( 2018 Ishii T, et al. This is an open-access article distributed under the terms of the Creative Commons Attribution License, which permits unrestricted use, distribution, and reproduction in any medium, provided the original author and source are credited 
Citation: Ishii T, Morimoto T, Shiraishi M, Kigawa Y, Narita K, et al. (2018) Retrospective Study of Trazodone Monotherapy Compared with Ramelteon and Trazodone Combination Therapy for the Management of Delirium. J Psychiatry 21: 444. doi:10.4172/2378-5756.1000444

Page 2 of 5

and secondly, to compare the efficacy of these two strategies as psychopharmacotherapy options for the management of delirium symptoms.

\section{Methods}

\section{Setting and participants}

This retrospective study was conducted at Sapporo Medical University Hospital in Sapporo, Japan. The study subjects were selected from 442 patients that attended the department of neuropsychiatry and were diagnosed with delirium between April 2013 and May 2016. All subjects were diagnosed using the ICD-10 criteria. Patients with alcohol or drug withdrawal syndromes were excluded because these conditions have different etiology from delirium caused by systemic disease. A total of 92 patients were extracted for the study that had been treated with trazodone and/or ramelteon but not co-medication of other psychoactive drugs. Of them, 33 patients were in the trazodone monotherapy group ( $\mathrm{T}$ group) and 59 patients in the ramelteon and trazodone combination therapy group (RT group). Patients' medical records were reviewed and the following data were extracted: age, gender, preexisting dementia, admission diagnosis, probable causes of delirium, dosage of the study drugs, and delirium severity.

\section{Measurement}

Delirium severity was measured using the Japanese version of the Delirium Rating Scale (DRS)-R-98 [22,23] and the Clinical Global Impression scale [24]. We collected the following data from the medical records: DRS-R-98 score and Clinical Global ImpressionSeverity (CGI-S) score at the initial psychiatric examination prior to the administration of study drugs as the baseline assessment, DRS-R-98 score, Clinical Global Impression-Improvement (CGI-I) score, and adverse drug reactions at 3-7 days after starting the study drugs as an endpoint assessment. Improvement in delirium was defined as a reduction in the DRS-R- 98 score from baseline of $50 \%$ or more or a DRS-R-98 score of 12 or less without relapse, in line with previous studies $[25,26]$.

All data were anonymously analysed without individual patient consent because of the retrospective design of the study. The Internal Review Board of Sapporo Medical University waived the need for individual informed consent and approved the study protocol. Only demographic data were obtained from the medical records of patients, and these data were collected prior to analysis in a manner whereby subjects could not be identified either directly or through identifiers linked to the subjects.

\section{Statistical analysis}

Group differences in patients' characteristics at baseline were examined by unpaired $t$-test or chi-square test. Comparisons between the groups were performed using two-way repeated measures ANOVA, with time (baseline vs. endpoint) and treatment group (T group vs. RT group) as independent factors and the DRS-R-98 score as a dependent variable. In addition, a chi-square test was used to examine differences in the proportion of patients who met the improvement criteria. Finally, an unpaired $t$-test was used to examine the group differences in CGI-I scores. All statistical analyses were performed using the SPSS Statistics software package, version 21.0 (SPSS Inc., Chicago, IL, USA), with the level of significance set at 0.05 .

\section{Results}

Participants' characteristics at baseline are shown in Table 1.

\begin{tabular}{|c|c|c|c|}
\hline \multirow{2}{*}{ Characteristic } & T group & RT group & \multirow{2}{*}{ p-value } \\
\hline & $(n=33)$ & $(n=59)$ & \\
\hline Age, mean (SD), y & $76.6(9.8)$ & $74.6(10.4)$ & 0.359 \\
\hline Male sex, No. (\%) & $22(67)$ & $33(56)$ & 0.314 \\
\hline Dementia, No. (\%) & $11(33)$ & $18(31)$ & 0.780 \\
\hline \multicolumn{4}{|l|}{ Admission diagnosis, No.(\%) } \\
\hline Cancer & $12(36)$ & $24(41)$ & 0.684 \\
\hline Heart failure / myocardial infarction & $7(21)$ & $11(19)$ & 0.766 \\
\hline Stroke & $4(12)$ & $11(19)$ & 0.417 \\
\hline Trauma & $4(12)$ & $13(22)$ & 0.240 \\
\hline Respiratory problem & $5(15)$ & $5(8)$ & 0.258 \\
\hline Autoimmune disease & $1(3)$ & $5(8)$ & 0.295 \\
\hline Other & $9(27)$ & $10(17)$ & 0.241 \\
\hline \multicolumn{4}{|l|}{ Cause of delirium, No.(\%) } \\
\hline Postoperative & $14(42)$ & $28(47)$ & 0.642 \\
\hline Infection & $13(39)$ & $19(32)$ & 0.487 \\
\hline Electrolyte & $7(21)$ & $8(14)$ & 0.341 \\
\hline Trauma & $3(9)$ & $12(20)$ & 0.161 \\
\hline CNS & $9(27)$ & $19(32)$ & 0.622 \\
\hline Hypoxic & $3(9)$ & $5(8)$ & 0.600 \\
\hline Organ failure & $5(15)$ & $6(10)$ & 0.348 \\
\hline Incretion & $1(3)$ & $0(0)$ & 0.359 \\
\hline Hemodynamics & $2(6)$ & $9(15)$ & 0.167 \\
\hline Drug induced & $19(58)$ & $29(49)$ & 0.438 \\
\hline Other & $4(12)$ & $9(4)$ & 0.468 \\
\hline Total $\mathrm{n}$. cause of delirium, mean (SD) & $2.4(1.2)$ & $2.5(1.2)$ & 0.694 \\
\hline \multicolumn{4}{|l|}{$\begin{array}{l}\text { Medication dose of study drug, mean } \\
\text { (SD), mg }\end{array}$} \\
\hline Trazodone & $35.6(20.5)$ & $32.6(17.1)$ & 0.452 \\
\hline Ramelteon & 0 & $7.8(0.9)$ & - \\
\hline CGI-S score, mean (SD) & $4.1(1.0)$ & $4.4(1.0)$ & 0.078 \\
\hline
\end{tabular}

Abbreviations: T group-trazodone monotherapy group; RT group-ramelteon and trazodone combination therapy group: SD-standard deviation; CNS-Centra Nervous System; CGI-S-Clinical Global Impression-Severity

Table 1: Baseline patient characteristics.

There were no significant differences in demographic characteristics, proportion of pre-existing dementia and other admission diagnoses, cause of delirium, dose of trazodone and CGI-S score at baseline between the groups.

At baseline, mean \pm SD DRS-R-98 score was $22.0 \pm 5.5$ in the T group and $23.7 \pm 6.1$ in the RT group. Following treatment, the mean \pm SD DRS-R-98 score was $13.5 \pm 8.5$ in the T group and $11.4 \pm 8.6$ in the RT group. Two-way repeated measures ANOVA showed that there was a significant effect of time $(F[1,90]=95.632, \mathrm{p}<0.001)$ on the total DRS-R-98 score (Figure 1a). However, no effect of treatment group $(\mathrm{F}[1,90]=0.259, \mathrm{p}=0.612)$ or time $\times$ treatment group interaction $(\mathrm{F}[1$, $90]=1.111, p=0.295)$ was observed on the total DRS-R-98 score. These effects were similar to the DRS-R-98 non-cognitive (1-8) and cognitive (9-13) subscale scores (Figures $1 \mathrm{~b}$ and 1c). Significant effects of time were observed ( $1-8$ sum score: $F[1,90]=90.606, p<0.001 ; 9-13$ sum score: $\mathrm{F}[1,90]=72.121, \mathrm{p}<0.001)$, but no effects of treatment group $(1-8$ sum score: $\mathrm{F}[1,90]=0.024, \mathrm{p}=0.876 ; 9-13$ sum score: $\mathrm{F}[1,90]=0.705$, $\mathrm{p}=0.403)$ and time $\times$ treatment group interaction (1-8 sum score: $\mathrm{F}[1$, $90]=1.226, \mathrm{p}=0.271 ; 9-13$ sum score: $\mathrm{F}[1,90]=0.633, \mathrm{p}=0.428)$.

Notably, the proportion of patients who met the remission criterion of improvement in total DRS-R-98 score was significantly higher in the RT group compared with the T group ( $71 \%$ vs. $48 \%$; chi-square $=4.681$, 
Page 3 of 5

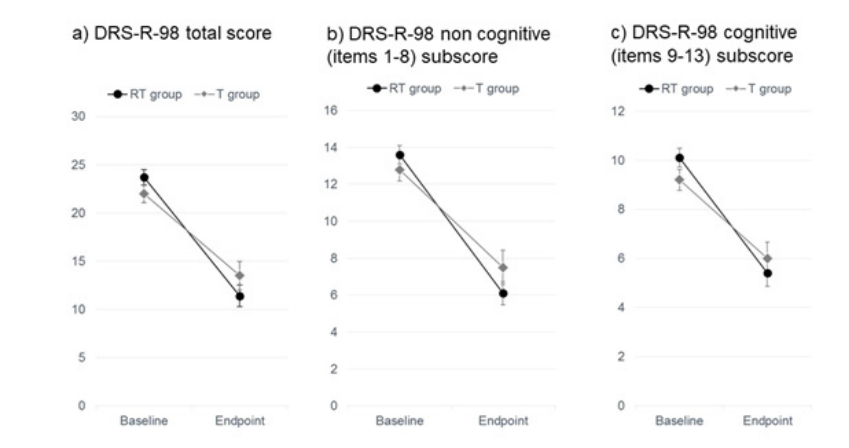

Note: DRS-R-98-Delirium Rating Scale-Revised-98; T group-trazodone monotherapy group; RT group- ramelteon and trazodone combination therapy group

Figure 1: Mean difference in DRS-R-98 severity scores from baseline to the endpoint.

$\mathrm{p}=0.030$ ). Moreover, the mean \pm SD of the CGI-I scores was $2.7 \pm 1.3$ in the $\mathrm{T}$ group and $2.1 \pm 1.1$ in the RT group, which was shown by unpaired $t$-test to be significantly different between the groups $(\mathrm{t}=2.575$, $\mathrm{df}=90, \mathrm{p}=0.012)($ Table 2$)$.

A summary of adverse drug reactions attributable to each treatment is shown in Table 2. No critical adverse drug reactions were reported in either group. The most frequent adverse drug reaction was somnolence, in the RT group (3\%). Worsening of delirium symptoms was observed in two patients (4\%) in the T group and in one patient (1\%) in the RT group. In all cases, however, deterioration in the primary disease was observed by clinicians. There were no significant differences in the proportion of patients with worsening of delirium symptoms or who suffered from somnolence attributable to the study drugs.

\section{Discussion}

The present study implied that both trazodone monotherapy and ramelteon and trazodone combination therapy were effective and safe in controlling delirium symptoms caused by multiple etiologies. In the combination therapy group, however, more patients met the total DRS-R-98 score improvement criteria. In addition, the mean CGI-I score was significantly lower, with more patients meeting the improvement criterion, in the combination therapy group, supporting the effectiveness of ramelteon and trazodone combination therapy for delirium. This study is, to our knowledge, the first to demonstrate the effects of trazodone monotherapy and ramelteon and trazodone combination therapy on the symptoms of delirium. Furthermore, no critical adverse drug reactions were reported in either group. Although several case reports have described delirium caused by trazodone in depressive patients $[27,28]$, we observed no worsening of delirium symptoms attributable to the study drug among patients in the present study.

A disturbed diurnal sleep-wake cycle involving sleep fragmentation may be a key factor in the pathogenesis of delirium [29]. Sleep-wake cycle adjustment in patients with delirium has been reported to improve the other symptoms of delirium such as emotional disturbances and behavioral changes [29]. Emerging evidence indicates that the pathophysiology of delirium is associated with a low melatonin level and the absence of a normal melatonin secretion rhythm [30,31]. Ramelteon demonstrates a high affinity for $\mathrm{MT}_{1}$ and $\mathrm{MT}_{2}$ melatonin receptors, which are located in the suprachiasmatic nucleus of the hypothalamus and are involved in maintaining the circadian sleep-

\begin{tabular}{|c|c|c|c|}
\hline \multirow{2}{*}{ Characteristics } & T group & RT group & \multirow{2}{*}{-value } \\
\cline { 2 - 4 } & $\mathbf{( n = 3 3 )}$ & $\mathbf{( n = 5 9 )}$ & \\
\hline Remission, No. (\%) & $16(48)$ & $42(71)$ & 0.03 \\
\hline CGI-I, mean (SD) & $2.7(1.3)$ & $2.1(1.1)$ & 0.012 \\
\hline Adverse drug events, No.(\%) & & & \\
\hline Somnolence & $0(0)$ & $2(3)$ & 0.409 \\
\hline Worsening of delirium symptoms & $2(4)$ & $1(1)$ & 0.292 \\
\hline Other & $0(0)$ & $0(0)$ & - \\
\hline
\end{tabular}

Abbreviations: T group-trazodone monotherapy group; RT group-ramelteon and trazodone combination therapy group; SD-standard deviation; CGI-I-Clinical Global Impression-Improvement

Table 2: Clinical outcomes and adverse drug reactions in the study drug groups.

wake cycle [32]. Ramelteon is a hypnotic that induces more natural sleep and has a safer but less effective sedative effect than other types of hypnotics $[33,34]$. Therefore, agitated-delirium patients treated with ramelteon frequently require additional hypnotics to induce sleep or control other symptoms of delirium. In fact, in a randomized, placebocontrolled study of the effectiveness of ramelteon for the prevention of delirium, $18 \%$ of patients treated with ramelteon used hydroxyzine as needed for insomnia [19].

Trazodone, a triazolepyridine derivate, is a heterocyclic antidepressant with strong antagonistic activity against 5-hydroxytryptamine-2 $\left(5-\mathrm{HT}_{2}\right)$ receptors as an acute pharmacologic effect [17]. In normal subjects, trazodone decreases stage 1 and 2 sleep while increasing slow-wave sleep, with relatively little effect on REM sleep by means of 5- $\mathrm{HT}_{2}$ receptor antagonism [35]. Difficulty in waking the next day is not typically associated with trazodone because of its short half-life [36]. Trazodone may also be effective in treating delirium because it decreases sleep-wake rhythm disturbance. Thus, ramelteon and trazodone have different mechanisms of action in improving sleepwake rhythm disturbance and may therefore synergistically control the symptoms of delirium.

Primary prevention using non-pharmacological multicomponent approaches is widely accepted as the most effective strategy for delirium [2]. At the same time, evidence for the efficacy and safety of antipsychotic use in patients with delirium has been accumulating $[11,37]$. In a recent prospective study in a cancer treatment setting, more than $60 \%$ of patients managed with antipsychotics showed resolution of delirium [14]. In another recent double-blinded, randomized clinical trial in a general hospital setting, $75.0 \%$ of quetiapine-managed patients and $67.9 \%$ of haloperidol-managed patients achieved the remission criterion, defined as a reduction in DRS-R-98 score of 12 or less without relapse [26]. Although simple comparisons are less accurate, the findings of this study suggest that the efficacy rates of the study medications were similar to those observed previous studies on antipsychotics.

The U.S. Food and Drug Administration has warned that the treatment of elderly patients with atypical antipsychotics can lead to serious adverse drug reactions and was associated with increased mortality in 2005 [38]. They extended this warning to typical antipsychotic medications on the basis of further research in 2008 [38]. Since then, clinicians have hesitated to prescribe antipsychotics to older patients, despite the controversy associated with these warnings [38]. Extrapyramidal symptoms caused by antipsychotic medications are also a major concern in the pharmacotherapy of delirium. A 
Citation: Ishii T, Morimoto T, Shiraishi M, Kigawa Y, Narita K, et al. (2018) Retrospective Study of Trazodone Monotherapy Compared with Ramelteon and Trazodone Combination Therapy for the Management of Delirium. J Psychiatry 21: 444. doi:10.4172/2378-5756.1000444

Page 4 of 5

daily dose of $1.2-15 \mathrm{mg}$ of haloperidol may cause extrapyramidal symptoms in $19 \%-30 \%$ of patients with delirium $[14,39,40]$. In this study, extrapyramidal symptoms were not observed in either group, probably because ramelteon and trazodone are not dopamine receptor antagonists. Sedation or somnolence is also commonly associated with antipsychotics and other hypnotics. Sedation was reported in $17.3 \%-28.6 \%$ of haloperidol $(0.5-2.0 \mathrm{mg} /$ day)-managed patients with delirium and $41.7 \%$ of quetiapine $(25.5-100 \mathrm{mg} /$ day)-managed patients $[14,26,39,41]$. In this study, somnolence was observed, but at a very low incidence rate. Antipsychotic medications are frequently accompanied by adverse drug reactions and mortality, as mentioned above. The feasibility of using therapeutic alternatives such as trazodone and ramelteon for the management of delirium symptoms is therefore important.

The present study had several limitations. First, it was a retrospective study and the number of cases was small, given that only one institute participated. Second, there is a possibility that antipsychotics were administered to patients with more severe delirium while ramelteon and/or trazodone were administered to patients with mild or moderate delirium because mean baseline CGI-S was 4.1 in the T group and 4.4 in the RT group, ranging between the classification of "moderately ill" and "markedly ill" according to CGI-S guidelines [24]. Therefore, the efficacy of ramelteon and/or trazodone in more severe delirium remains to be elucidated. Further investigations, particularly welldesigned controlled studies, are needed to confirm our findings.

\section{Conclusion}

Our analysis implied that both trazodone monotherapy and ramelteon and trazodone combination therapy were effective in managing the symptoms of delirium. In the ramelteon and trazodone combination therapy group, however, more patients met the total DRS-R-98 score improvement criterion. Of note, few patients reported adverse drug reactions in either group. Our results support the use of therapeutic alternatives to antipsychotics for controlling less severe delirium symptoms, particular to minimize adverse drug reactions.

\section{Acknowledgments}

We thank Clare Cox, PhD, from Edanz Group (www.edanzediting.com/ac) for editing a draft of this manuscript. This research is supported by the Department of Neuropsychiatry alumni association, Sapporo Medical University.

\section{Disclosure}

The authors report no conflicts of interest in this work.

\section{References}

1. Siddiqi N, House AO, Holmes JD (2006) Occurrence and outcome of delirium in medical in-patients: a systematic literature review. Age Ageing 35: 350-364.

2. Inouye SK, Westendorp RG, Saczynski JS (2014) Delirium in elderly people. Lancet 383: 911-922.

3. Salluh JIF, Wang H, Schneider EB, Nagaraja N, Yenokyan G, et al. (2015) Outcome of delirium in critically ill patients: systematic review and metaanalysis. BMJ 350: h2538.

4. Buurman BM, Hoogerduijn JG, de Haan RJ, Abu-Hanna A, Lagaay AM, et al. (2011) Geriatric conditions in acutely hospitalized older patients: prevalence and one-year survival and functional decline. PLoS One 6: e26951.

5. Gaudreau JD, Gagnon P (2005) Psychotogenic drugs and delirium pathogenesis: the central role of the thalamus. Med Hypotheses 64: 471-475.

6. Flacker JM, Lipsitz LA (1999) Neural mechanisms of delirium: current hypotheses and evolving concepts. J Gerontol A Biol Sci Med Sci 54: B239246.

7. Maldonado JR (2013) Neuropathogenesis of delirium: review of current etiologic theories and common pathways. Am J Geriatr Psychiatry 21: 1190-1222.

8. Trzepacz P, Breitbart W, Franklin J, Levenson J, Martini J, et al. (1999) Practice guideline for the treatment of patients with delirium. Am J Psychiatry 156: 1-20.

9. Young J, Murthy L, Westby M, Akunne A, O'Mahony R (2010) Diagnosis prevention, and management of delirium: summary of NICE guidance. BM 341: c3704.

10. Neufeld KJ, Yue J, Robinson TN, Inouye SK, Needham DM (2016) Antipsychotic medication for prevention and treatment of delirium in hospitalized adults: A systematic review and meta-analysis. J Am Geriatr Soc 64: 705-714.

11. Lonergan E, Britton AM, Luxenberg J, Wyller T (2007) Antipsychotics for delirium. Cochrane Database Syst Rev CD005594.

12. Seitz DP, Gill SS (2009) Neuroleptic malignant syndrome complicating antipsychotic treatment of delirium or agitation in medical and surgical patients: case reports and a review of the literature. Psychosomatics 50: 8-15.

13. Hassaballa HA, Balk RA (2003) Torsade de pointes associated with the administration of intravenous haloperidol:a review of the literature and practical guidelines for use. Expert Opin Drug Saf 2: 543-547.

14. Boettger S, Jenewein J, Breitbart W (2015) Haloperidol, risperidone, olanzapine and aripiprazole in the management of delirium: A comparison of efficacy, safety, and side effects. Palliat Support Care 13: 1079-1085.

15. Lacasse H, Perreault MM, Williamson DR (2006) Systematic review of antipsychotics for the treatment of hospital-associated delirium in medically or surgically ill patients. Ann Pharmacother 40: 1966-1973.

16. Schroeck JL, Ford J, Conway EL, Kurtzhalts KE, Gee ME, et al. (2016) Review of Safety and Efficacy of Sleep Medicines in Older Adults. Clin Ther 38: 23402372 .

17. James SP, Mendelson WB (2004) The use of trazodone as a hypnotic: a critical review. J Clin Psychiatry 65: 752-755.

18. Okamoto Y, Matsuoka Y, Sasaki T, Jitsuiki H, Horiguchi J, et al. (1999) Trazodone in the treatment of delirium. J Clin Psychopharmacol 19: 280-282.

19. Hatta K, Kishi Y, Wada K, Takeuchi T, Odawara T, et al. (2014) Preventive effects of ramelteon on delirium: a randomized placebo-controlled trial. JAMA Psychiatry 71: 397-403.

20. Tsuda A, Nishimura K, Naganawa E, Otsubo T, Ishigooka J (2014) Ramelteon for the treatment of delirium in elderly patients: a consecutive case series study. Int J Psychiatry Med 47: 97-104.

21. Miyata R, Omasa M, Fujimoto R, Ishikawa H, Aoki M (2017) Efficacy of Ramelteon for delirium after lung cancer surgery. Interact Cardiovasc Thorac Surg 24: 8-12.

22. Trzepacz PT, Mittal D, Torres R, Kanary K, Norton J, et al. (2001) Validation of the Delirium Rating Scale-revised-98: comparison with the delirium rating scale and the cognitive test for delirium. J Neuropsychiatry Clin Neurosci 13 229-242.

23. Kato M, Kishi Y, Okuyama T, Trzepacz PT, Hosaka T (2010) Japanese version of the Delirium Rating Scale, Revised-98 (DRS-R98-J): reliability and validity. Psychosomatics 51: 425-431.

24. Guy W (1976) ECDEU Assessment Manual for PsychopharmacologyRevised. U.S. Department of Health, Education, and Welfare, Rockville, MD.

25. Kimura R, Mori K, Kumazaki H, Yanagida M, Taguchi S, et al. (2011) Treatment of delirium with ramelteon: initial experience in three patients. Gen Hosp Psychiatry 33: 407-409.

26. Maneeton B, Maneeton N, Srisurapanont M, Chittawatanarat K (2013) Quetiapine versus haloperidol in the treatment of delirium: a double-blind randomized, controlled trial. Drug Des Devel Ther 7: 657-667.

27. Damlouji NF, Ferguson JM (1984) Trazodone-induced delirium in bulimic patients. Am J Psychiatry 141: 434-435

28. Lennkh C, Fischer P, Kufferle B, Kasper S (1998) Occurrence of trazodoneinduced delirium. Int Clin Psychopharmacol 13: 225-228.

29. Lipowski ZJ (1987) Delirium (acute confusional states). JAMA 258: 1789-1792.

30. Olofsson K, Alling C, Lundberg D, Malmros C (2004) Abolished circadian rhythm of melatonin secretion in sedated and artificially ventilated intensive care patients. Acta Anaesthesiol Scand 48: 679-684. 
Citation: Ishii T, Morimoto T, Shiraishi M, Kigawa Y, Narita K, et al. (2018) Retrospective Study of Trazodone Monotherapy Compared with Ramelteon and Trazodone Combination Therapy for the Management of Delirium. J Psychiatry 21: 444. doi:10.4172/2378-5756.1000444

Page 5 of 5

31. Bellapart J, Boots R (2012) Potential use of melatonin in sleep and delirium in the critically ill. Br J Anaesth 108: 572-580.

32. Brzezinski A (1997) Melatonin in humans. N Engl J Med 336: 186-195.

33. Griffiths RR, Johnson MW (2005) Relative abuse liability of hypnotic drugs: a conceptual framework and algorithm for differentiating among compounds. J Clin Psychiatry 66: 31-41.

34. Johnson MW, Suess PE, Griffiths RR (2006) Ramelteon: a novel hypnotic lacking abuse liability and sedative adverse effects. Arch Gen Psychiatry 63: 1149-1157.

35. Yamadera H, Nakamura S, Suzuki H, Endo S (1998) Effects of trazodone hydrochloride and imipramine on polysomnography in healthy subjects. Psychiatry Clin Neurosci 52: 439-443.

36. Fabre LF (1990) Trazodone dosing regimen: experience with single daily administration. J Clin Psychiatry 51: 23-26.

37. Hatta K, Kishi Y, Wada K, Odawara T, Takeuchi T, et al. (2014) Antipsychotics for delirium in the general hospital setting in consecutive 2453 inpatients: a prospective observational study. Int J Geriatr Psychiatry 29: 253-262.

38. Dorsey ER, Rabbani A, Gallagher SA, Conti RM, Alexander GC (2010) Impact of FDA black box advisory on antipsychotic medication use. Arch Intern Med 170: 96-103.

39. Yoon HJ, Park KM, Choi WJ, Choi SH, Park JY, et al. (2013) Efficacy and safety of haloperidol versus atypical antipsychotic medications in the treatment of delirium. BMC Psychiatry 13: 240.

40. Rea RS, Battistone S, Fong JJ, Devlin JW (2007) Atypical antipsychotics versus haloperidol for treatment of delirium in acutely ill patients. Pharmacotherapy 27 588-594.

41. Kim SW, Yoo JA, Lee SY, Kim SY, Bae KY, et al. (2010) Risperidone versus olanzapine for the treatment of delirium. Hum Psychopharmacol 25: 298-302. 